\section{Belimumab and the measurement of fatigue}

I read with great interest the article by Strand $e t a l .{ }^{1}$ The aim of the study was to assess the effects of belimumab treatment plus standard systemic lupus erythematosus (SLE) therapy on health-related quality of life in patients with active, autoantibody-positive SLE. Of particular interest are the results about the impact of belimumab on fatigue scores.

Fatigue is a common complaint of patients with SLE and is associated with diminished ability to function. ${ }^{2}{ }^{3}$ In the majority of cases, the cause of fatigue is still unexplained and it is likely that many factors together lead to lupus fatigue. ${ }^{2}$ However, until recently, clinical trials have not routinely assessed fatigue. ${ }^{3}$

The article by Strand $e t ~ a l^{1}$ showed that belimumab treatment can partially reduce fatigue, measured through the Functional Assessment of Chronic Illness Therapy-Fatigue Scale (FACIT-F scale). ${ }^{4}$ In more detail, FACIT-F scores were not significantly different across treatment groups at the week 24 prespecified secondary endpoint. However, scores from baseline to week 52 improved significantly $(\mathrm{p}<0.05)$ with belimumab 1 and $10 \mathrm{mg} / \mathrm{kg}$ versus placebo in A Study of Belimumab in Subjects with Systemic Lupus Erythematosus (BLISS)-52, and with $1 \mathrm{mg} / \mathrm{kg}$ at weeks 52 and 76 secondary endpoints in BLISS-76. Differences observed between belimumab $10 \mathrm{mg} / \mathrm{kg}$ and placebo were not statistically significant in BLISS-76, but numerical improvements over placebo were observed by week 8 and sustained through week $76{ }^{1}$

The BLISS trials adopted the FACIT-F as one of the outcome measures. This scale is a 13 -item questionnaire that assesses selfreported fatigue and its effect upon daily activities and function. It was originally developed for the precise evaluation of fatigue associated with anaemia in patients with cancer. Subsequently, it has been employed in many diseases, including patients with rheumatoid arthritis ${ }^{4}$ and psoriatic arthritis. ${ }^{5}$

FACIT-F is usually considered a unidimensional scale, with high internal reliability. However, in a recent study, published after the design of BLISS trials, the factorial structure of the FACIT-F has been questioned. More precisely, in the work by Cella $e t a l,{ }^{6}$ the 13 items were classified into either 'experience' (five items) or 'impact' (eight items). Experience items inquire about perceptions and severity of feeling states, including tiredness, energy level, weakness, fatigue and listlessness. Impact items inquire as to whether fatigue (or tiredness) has had an impact upon an individual's daily functioning such as performing usual activities, social activity, sleep and eating. Item classification was done independently by three investigators with $100 \%$ agreement, and these classifications were confirmed in subsequent meetings with external groups including investigators from the pharmaceutical industry and the US Food and Drug Administration. Moreover, experience scores, while highly correlated to impact scores, appeared to be systematically lower (worse) than impact scores, across the samples. The authors concluded that, even if it is valid to report a single score, according to bifactor analysis, it would be valid if clinicians decide to report experience and impact scores separately. ${ }^{6}$ What I wonder here is whether Strand et al considered the opportunity to run analyses, separating these two fatigue dimensions. Moreover, I suggest that a further investigation on smaller groups of items (or single items), even if explorative and not strictly informative for treatment efficacy, would lead to a better understanding of the treatment effects. These results would be inspiring for both future research on belimumab and for the physiological and psychological processes that may contribute to fatigue during the course of SLE.

\section{Davide Mazzoni}

Correspondence to Dr Davide Mazzoni, Department of Psychology, University of Bologna, 47126 Cesena (FC), Italy; davide.mazzoni@unibo.it

Competing interests None declared.

Provenance and peer review Not commissioned; internally peer reviewed.

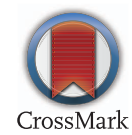

To cite Mazzoni D. Ann Rheum Dis 2015;74:e53.

Accepted 30 March 2015

Published Online First 10 April 2015

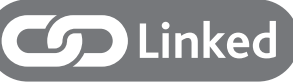

- http://dx.doi.org/10.1136/annrheumdis-2015-207700

Ann Rheum Dis 2015;74:e53. doi:10.1136/annrheumdis-2015-207693

\section{REFERENCES}

1 Strand V, Levy RA, Cervera R, et al. Improvements in health-related quality of life with belimumab, a B-lymphocyte stimulator-specific inhibitor, in patients with autoantibody-positive systemic lupus erythematosus from the randomised controlled BLISS trials. Ann Rheum Dis 2014;73:838-44.

2 Tench CM, McCurdie I, White PD, et al. The prevalence and associations of fatigue in systemic lupus erythematosus. Rheumatology 2000;39:1249-54.

3 Ramsey-Goldman R, Rothrock N. Fatigue in systemic lupus erythematosus and rheumatoid arthritis. PM R 2010;2:384-92.

4 Cella D, Yount S, Sorensen M, et al. Validation of the Functional Assessment of Chronic Illness Therapy Fatigue scale relative to other instrumentation in patients with rheumatoid arthritis. J Rheumatol 2005;32:811-19.

5 Chandran V, Bhella S, Schentag C. Functional assessment of chronic illness therapy-fatigue scale is valid in patients with psoriatic arthritis. Ann Rheum Dis 2007:66:936-9.

6 Cella D, Lai JS, Stone A. Self-reported fatigue: one dimension or more? Lessons from the Functional Assessment of Chronic Illness Therapy--Fatigue (FACIT-F) questionnaire. Support Care Cancer 2011;19:1441-50. 